the differential impact of the COVID-19 pandemic on the sleep and mental wellbeing of CYP with and without SEN.

Methods NIHR Children and Young People MedTech Co-operative, Sheffield Children's NHS Foundation Trust, and The Sleep Charity carried out an online survey between 23 June 2020 and 17 August 2020. The 77-item survey was shared via social media platforms.

Results 559 participants were included in the analyses, 15.74\% of whom reported having a CYP with SEN. While sleep changes due to the pandemic were largely similar for both groups, CYP with SEN (40.91\%) were more likely to get or wake up during the night than CYP without SEN (40.91\% vs 27.18\%). CYP with SEN were significantly more likely than children without SEN to be demotivated (61.44\% vs $31.57 \%)$, sad and tearful $(36.15 \%$ vs $19.35 \%)$ or anxious and stressed $(43.48 \%$ vs $14.82 \%)$ during the pandemic, as well as to report that increased anxiety was more likely to contribute to poorer sleep (43.48\% vs $14.82 \%)$.

Conclusion While the majority of CYP in both groups reported sleep changes due to the pandemic, CYP with SEN experienced more sleep disturbance. The findings provide initial evidence to suggest that the pandemic may have had a greater impact on the sleep and mental wellbeing of CYP with SEN compared to CYP without SEN.

\section{THE COMFORT PROJECT: DEVELOPMENT OF CUSTOM- MADE MASKS FOR CHILDREN USING NON-INVASIVE VENTILATION}

${ }^{1}$ Heather Elphick*, ${ }^{1}$ Nicki Barker, ${ }^{3}$ Peter Metherall, ${ }^{2}$ Matt Willox, ${ }^{3}$ Heath Reed, ${ }^{4}$ Katherine Jeays-Ward, ${ }^{4}$ Avril McCarthy. 'Sheffield Children's Hospital, Sheffield, UK; ${ }^{2}$ Sheffield Hallam University, Sheffield, UK; ${ }^{3}$ Sheffield Teaching Hospitals, Sheffield, UK; ${ }^{4}$ NIHR Devices 4 Dignity, Sheffield, UK

\subsection{6/bmjresp-2021-bssconf.26}

Non-invasive ventilation (NIV) is assisted respiratory support delivered via facemask for people with chronic respiratory failure. Commercial NIV masks are available but masks that fit well are difficult to find for children who have small or asymmetrical facial features. Compromised ventilation can have significant health and quality of life impacts for patients and their families.

The overarching aim of the project was to improve comfort, fit and performance of NIV masks for children using 3D technology.

The needs of patients, parents/carers and healthcare professionals from 6 UK centres was ensured through events advising on mask design and research procedures. 3D scanning technologies were compared in vitro using workflows and deviation analysis and with 3 healthy and 19 patient volunteers. Early design concepts were tested and the final
Abstract 29 Table 1 COMFORT project: development of custommade masks for children using non-invasive ventilation

\begin{tabular}{|l|l|l|}
\hline Oxygen parameters & Usual mask & Bespoke mask \\
\hline Median 02 & $95 \%$ & $98 \%$ \\
\hline Dip index & 20.4 & 8.0 \\
\hline Time $<90 \%$ & $5.8 \%$ & $1.1 \%$ \\
\hline Min 02 & $71 \%$ & $79 \%$ \\
\hline
\end{tabular}

prototype selected using a test mannequin and adult volunteers. Prototype masks were manufactured using biocompatible materials to IOS 13485 quality standards and evaluated with 19 patients. Feedback on fit, comfort and facial marking and oxicapnography data to demonstrate ventilator effectiveness were collected. The potential cost-effectiveness of the customised masks was assessed using an economic model.

A handheld structured light 3D scanner was selected due to superior resolution, ease of use, availability and cost. The final design was a semi-bespoke hybrid approach. 11/15 (73\%) evaluation participants reported comfort and fit that was as good or better than their usual mask. 5/11 (45\%) were unable to wear the mask due to poorly fitting headgear despite a well-fitting mask. All physiological ventilation parameters were improved (table 1). Preliminary health economics analysis demonstrated that to achieve a $5 \%$ improvement in fit and ventilation, a custom-made mask would be cost effective at a price below $£ 500$.

A series of in vitro, user-perspective, healthy volunteer and patient evaluations informed the development of custom-made masks for children using NIV.

\section{A COMPARISON OF MASIMO RAD97 AND SOMNOTOUCH OXIMETERS IN THE ASSESSMENT OF SLEEP DISORDERED BREATHING IN PAEDIATRIC PATIENTS}

Joe Madge, Natalie Blyth, Prakash Patel, David Luyt, Imad Ahmed. Univeristy Hospitals of Leicester Trust, Leicester, UK

\subsection{6/bmjresp-2021-bssconf.27}

Introduction Overnight oximetry is recommended as an initial screening tool to diagnose sleep disordered breathing (SDB) in children (Hang et al 2015). Oximetry is a key component of cardio-respiratory sleep studies (CRSS). Most CRSS equipment has integrated oximeters. This study aims to compare the oxygen saturation data obtained by the standalone Masimo Rad97 oximeter to the integrated Somnotouch oximeter.

Abstract 30 Table 1 A comparison of Masimo Rad97 and Somnotouch oximeters in the assessment of sleep disordered breathing in paediatric patients

\begin{tabular}{|l|l|l|l|}
\hline & Somnotouch RESP & Masimo rad 97 & -value \\
\hline Mean Oxygen Saturation & $96+/-3.9$ & $96.4+/-3.9$ & 0.007 \\
\hline ODI3 & $11.5+/-16.3$ & $18+/-27.1$ & 0.0006 \\
\hline Number of desaturations & $173.3+/-498.6$ & $149.2+/-239.1$ & 0.004 \\
\hline Time $\mathrm{SpO}_{2}=<90 \%$ & $5.3+/-19.4$ & $5.3+/-18.9$ & 0.85 \\
\hline
\end{tabular}

